\title{
Research on Application of Circuit Simulation Software in Electronic Technology Teaching
}

\author{
Lin Haifeng, Mao Ruili \\ BEIJING INFORMATION TECHNOLOGY COLLEGE
}

\begin{abstract}
Keywords: Application, Circuit Simulation Software, Electronic Technology Teaching
\end{abstract}
\begin{abstract}
Simulation technology makes the analysis of electronic circuits can be accurately and quickly completed in the computer. On the one hand it overcomes the limitations of laboratory components and instruments, on the other hand it also broke the time and space constraints. This paper describes the application methods of circuit simulation technology in the of electronic teaching, as well as its advantages in the e-learning, so that the students grasp this technology better to learn the knowledge of electronic technology better.
\end{abstract}

\section{Introduction}

Electronic technology-related courses are the important professional electronics students' basic professional courses. As the course content is abstract, strong theoretical, professional terminology, the concept of more nouns, students understand and accept the difficulties, thus losing interest in learning. The application of simulation technology in the teaching of electronic technology-related courses, so that the theory of teaching becomes vivid, image, real, credible, to stimulate students' strong desire for knowledge, so as to further improve their ability to design and innovation, analysis and problem-solving ability .

With the rapid development of computer technology, simulation technology has gradually become an important tool for circuit analysis and auxiliary design in the field of electronic engineering. Electronic technology is both the theory and practice of electric basic courses and in the electronic teaching system has a pivotal role. The traditional teaching of electronic technology is the theory of teaching and experiment separately, so to explain the more abstract theory, the teacher draw the circuit diagram, analysis of the working principle of the circuit, derivation formula, the circuit performance parameters, but the lack of intuitive impression, Students do not understand the content of the course, the teaching effect is often not ideal. If we use circuit simulation software to analyze the performance parameters of circuit quickly, we can make the abstract theory be tested in time and give the students a more intuitive understanding. The teaching effect will be greatly improved. At the same time, the simulation can also show the practical effect of circuit work, help students understand and analyze complex circuits.

\section{The Introduction of Circuit Simulation Software}

At present, the commonly used circuit simulation software can be divided into two categories, all with the features as analog, digital and analog mixed-signal simulation. One is Spice as the core of the circuit simulation software, such as O1CAD suite of PSpice software, has a rich library of components to complete the circuit DC characteristics analysis, AC characteristics analysis and transient analysis and other basic analysis functions, quickly and accurately calculate Complex circuit performance parameters, the text or waveform curve gives the analysis results. PSpice also features parametric sweep analysis to analyze the effect of component variations on circuit performance, and Monte Carlo and worst case analysis to simulate the actual dispersion of component parameters in real production the dispersion of circuit characteristics, the dispersion of the circuit characteristics of the law. The other is the virtual electronic workbench EWB (Electronics Work bench referred to) and its upgraded software Multisim, the biggest feature of such software is the use of computers to construct a virtual experimental environment, with a variety of circuit 
analysis and design of the electronic Instruments such as digital multimeters, oscilloscopes, spectrum analyzers, function generators, etc., and with light-emitting diodes and digital tubes and other display devices, can be an intuitive demonstration of the actual work of the circuit.

\section{The Role of EDA Technology in the Electronic Technology Teaching}

The introduction of EDA into electronic technology practice teaching is an important content of teaching reform in the new period. It plays an important role in breaking the traditional teaching mode, reforming teaching content, innovating teaching methods and consolidating teaching objectives. With the extensive application of EDA in the teaching of electronic technology, it has more obvious advantages in teaching, mainly reflected in the following aspects:

The electronic technology teaching is a very practical subject, and it has a strong logic, students learn more boring and difficult to understand, students must rely on the experimental teaching intuitive and operable to consolidate theoretical knowledge. In the experimental teaching of electronic technology, students in addition to the master of some commonly used instruments to use methods and parameter measurement methods, but also through experiments to master the various components and various types of circuit performance. As for the complex circuit, it is difficult for students to grasp the circuit in a short time the performance and output targets, EDA has accurate simulation, and has a graphical and text output, which students a comprehensive understanding of the circuit helpful. Therefore, EDA can effectively improve the quality of electronic technology practice teaching.

Electronic technology is a strong theoretical course and if the students should not be learning methods, it is easy to lose interest in learning. However, in the teaching, the non-intellectual factors such as learning motivation, learning interest, emotion and perseverance have a direct impact on the teaching effect. Therefore, in the curriculum reform of electronic technology, new teaching methods and means must consider these non-intellectual factors effect. Compared with the traditional teaching methods and teaching methods, EDA technology has the advantages of intuitionistic, lively and interesting in classroom teaching. EDA technology into practical teaching can make the objective things macroscopic expression, can make the abstract concept image expression, and can help students to verify the theoretical knowledge, understand the complex circuit change process, so as to achieve the combination of theory and practice effect.

Higher vocational education wants to improve the practical ability of students and innovative design capabilities and we must resort to advanced teaching methods and teaching facilities to meet the teaching needs. In the practical sense, the virtual laboratory is an indispensable part of the higher vocational colleges to realize the simulation teaching. At the same time, the virtual laboratory can also alleviate the expense of experiment and practice in higher vocational colleges. It is a good combination of theory and practice. s method. Compared with traditional experimental teaching methods, EDA teaching laboratory has the features of full function, low cost, high efficiency, portability and engineering practicability. It overcomes the limitations of traditional laboratory instruments in varieties, specifications and quantity, Can be implemented design, test, validation and error correction, innovative and other experiments.

Electronics is a very experimental subject, the need to synchronize the theory and experiment. The measurement equipment and electronic components required by the experiment are often limited by the objective conditions, and the aging of the old equipment in the laboratory of the most vocational institutions is not possible to provide the latest devices of the manufacturers in the world. The use of circuit simulation technology can provide a variety of new device simulation model, the virtual device not only looks realistic, and has a real device functions, the user is like entering a real advanced laboratory.

In electronic experiments, sometimes we will encounter some cases, is due to experimental equipment failure and lead to data errors, or we want to analyze a component failure on the circuit have any effect, in theory, we can analyze the data at this time the results, but more difficult to use real experiments to simulate these fault conditions. For example, some electronic designs involve 
high voltage and high current. Incorrect design parameters may cause component damage and block the design. Therefore, using simulation techniques to test the design prior to building a real circuit can avoid a variety of fatal injuries.

Use the circuit simulation technology you can remove a lot of trouble, such as: preparation of experimental materials and equipment before the experiment, the experiment Pin inserted, welding experimental components and the use of laboratory equipment, student safety, Experimental materials, and routine maintenance. These jobs often cost a lot of time, manpower and financial resources. We can put all the energy used in doing simulation experiments above, and no matter how students do the experimental simulation, components of any components and test equipment will not be damaged or lost. Practice has proved that the use of circuit simulation technology, in the virtual laboratory to do experiments, greatly improve the efficiency of experiments, and virtual experimental system easy to use, easy to self-study.

In the virtual laboratory to achieve the "software that is the instrument" software components ", so fundamentally solve the shortage of funds, improper use, mismanagement and many other factors on the experiment Teaching and practice of teaching a serious constraint. It will not be the same as the actual operation of the component multiple soldering and damage to the device and printed circuit board, the circuit debugging fast and convenient, can make the circuit to achieve the best condition. At the same time simulation software provides performance Excellent virtual instrument and realistic testing environment, can easily observe the general instrument inconvenience or cannot observe the waveform can be directly printed output of experimental data, test curves and circuit schematics, the effect is excellent due to the rich virtual electronic element Devices and virtual instrumentation, from the fundamental to overcome the laboratory instruments in terms of variety, size, number of restrictions, you can achieve in the virtual laboratory verification, test, design, error correction, innovative and so on As long as we have a computer plus a set of electronic simulation software coupled with a virtual experiment tutorial, we are equivalent to have a sophisticated electronic laboratory equipment.

\section{The Basic Methods of Using Simulation Software for Circuit Analysis}

Application of PSpice circuit simulation analysis can be divided into four basic steps. Drawing the correct circuit is the basic requirement for simulation analysis, setting the simulation type and parameters is the key step of circuit simulation analysis. Whether the simulation analysis and can get intuitive simulation results and simulation type and parameter settings are closely related. Application of Multisim circuit analysis and simulation of the basic process is similar to PSpice, but also in the drawing circuit diagram also includes the necessary equipment to form a complete experimental circuit. In the digital circuit simulation or analog circuit transient analysis, do not need to set the simulation type and parameters, only need to open the virtual power switch to simulate and display the simulation results.

\section{The Application of Circuit Simulation Software in the Electronic Technology Teaching}

The application of circuit simulation software to assist theoretical teaching, the theory in time to be verified in the simulation, teaching a more vivid image, enhance the perceptual knowledge of students will undoubtedly improve the teaching effect. For example, to explain the sinusoidal oscillation circuit shown in Figure 2 (supply voltage 12V) according to theoretical analysis, in R2 + R3 $>2 \mathrm{R} 1$, to meet the starting conditions, the oscillator will output sine wave signal. Therefore, in the simulation process R3 by 5 values for simulation analysis. From the PSpice simulation results can be seen that the circuit only R2 + R3> 2R1 will produce oscillation, and the output amplitude of the sine wave signal amplitude and R3 have a close relationship, thus confirming the R3 and diode D1, D2 Of the role of stable links.

Virtual electronic technology experiment is the use of computer construction of an experimental environment, through the establishment of the circuit and the circuit function analysis, to achieve the 
experimental results and purpose of experimental methods. Usually, the components provided by the simulation software include transistors, resistive-capacitive components, integrated circuits and digital gate circuits. The instrument library includes oscilloscopes, signal generators, sweepers, logic analyzers, digital Signal generator and other equipment, connected to the switch can be carried out and the physical test of the same test and analysis.

As a complement to the traditional experimental methods, the virtual electronic technology experiment can inherit the advantages of operability and participation in physical experiment. On the other hand, it can make full use of computer advantage and share resources.

Traditional electronic technology curriculum design is based on the analog circuit and digital circuit theory, according to certain design requirements to achieve a relatively complete with a certain function and scale of the circuit. Whether the designed circuit can meet the pre-set functional requirements or pre-set technical indicators, often rely on teachers to check one by one, inevitably affected by the teacher's ability and energy, errors or omissions, so that curriculum design effects are affected. If you do before the course design, teachers will use the commonly used method of circuit simulation to teach students, students do not have to prepare components, do not build circuits, as long as the way through the simulation of their own design to check whether the circuit requirements, and may at any time modify Circuit structure and parameters until the design goal, and do not have to worry about damage to the instrument. Simulation design allows students to complete the course design independently, thus a more profound understanding of the design of the circuit, after the completion of a higher sense of accomplishment, for the future study has far-reaching impact. Therefore, compared with the traditional curriculum design methods, the effect is much better.

\section{Conclusion}

Using the simulation software to assist the of electronic technology courses teaching, so that the theory be verified at any time. Using the simulation software to carry on the experiment of the virtual electronic technology breaks the limitation in time and space of the traditional experiment, and we can arrange the experiment time flexibly according to their own hobbies and needs. Master the circuit simulation software is also the effective tools to lay a solid foundation for electronic system analysis and design for future work.

\section{References}

[1] X. Y. Zeng. .Application of EDA technology in digital circuit teaching, J. Experimental Technology and Management, 2006, 23 (5): 87-89

[2] J. T. Zhang. Application of PSpice virtual electronic technology experiments, J. Modern electronic technology, 2002 (2): 58-61.

[3] Y.X. Zhao. Electronic circuit PSPICE analysis and design, M. Tianjin: Tianjin University Press, 1999.

[4] Z.W. Huang. Based on Multisim 2001 electronic circuit simulation and design of computer analysis, M. Beijing: Electronic Industry Press, 2004

[5] Y.Q. Zhang. Design of electrical and electronic experiments using $\mathrm{O}_{1}$ CAD9.2, J. Experiment Technology and Management, 2006, 23 (9): 80-82 